\title{
Development and validation of a prognostic prediction model for antithrombotic-related chronic subdural hematoma in patients with recent acute myocardial infarction
}

\author{
Peng Zhou ${ }^{1,2 \#}$, Jindong Wan ${ }^{1,2 \#}$, Fei Ran ${ }^{1,2}$, Feng Gao ${ }^{3}$, Dachun Yang ${ }^{4}$, Xiaozhen Dai ${ }^{5}$, Yun Sun ${ }^{6}$, \\ Peijian Wang ${ }^{1,2}$
}

${ }^{1}$ Department of Cardiology, The First Affiliated Hospital, Chengdu Medical College, Chengdu, China; ${ }^{2}$ Key Laboratory of Aging and Vascular Homeostasis of Sichuan Higher Education Institutes, Chengdu, China; ${ }^{3}$ Department of Cardiology, The Second Hospital of Anhui Medical University, Hefei, China; ${ }^{4}$ Department of Cardiology, The General Hospital of Western Theater Command, Chengdu, China; ${ }^{5}$ School of Biosciences and Technology, Chengdu Medical College, Chengdu, China; ${ }^{6}$ Department of Party Secretary, The First Affiliated Hospital, Chengdu Medical College, Chengdu, China

Contributions: (I) Conception and design: P Zhou, P Wang, Y Sun; (II) Administrative support: F Gao, D Yang, X Dai, Y Sun; (III) Provision of study materials or patients: F Ran, F Gao, D Yang; (IV) Collection and assembly of data: J Wan, F Ran, F Gao, D Yang; (V) Data analysis and interpretation: P Zhou, P Wang, J Wan; (VI) Manuscript writing: All authors; (VII) Final approval of manuscript: All authors.

\#These authors contributed equally to this work.

Correspondence to: Peijian Wang, MD, PhD. Department of Cardiology, The First Affiliated Hospital of Chengdu Medical College, 278 Baoguang Avenue, Xindu District, Chengdu 610500, China. Email: wpjmed@aliyun.com; Yun Sun, MD. The First Affiliated Hospital of Chengdu Medical College, 278 Baoguang Avenue, Xindu District, Chengdu, China. Email: doctor_yun@sina.cn.

Background: Antithrombotic therapy is a cornerstone of acute myocardial infarction (AMI) treatment and is thought to be associated with an increased risk of chronic subdural hematoma (CSDH). However, no wellestablished model exists to predict subsequent antithrombotic treatment outcomes after CSDH in patients with recent AMI. We aimed to identify a prognostic model to predict the 6-month outcome of treatment with antithrombotic therapy.

Methods: This multicenter retrospective analysis involved 553 patients with recent AMI with antithrombotic-related CSDH. Several candidate clinical variables and biomarkers were examined in the training cohort (Chengdu training cohort; $n=368$ ). Patients with unfavorable outcomes had experienced at least 1 of the following: major adverse cardiovascular events (MACE), recurrence, or a modified Rankin scale (mRS) score of 2 to 6 . To develop a 6-month outcome prediction model, three approaches were used: (I) a demographic variable model, (II) a clinical marker model and (III) a decision-driven model. A clinical outcome prediction model based on the superior predictors was assessed by logistic regression analysis. The nomogram for the final model was internally validated using a bootstrap procedure and externally validated in an independent cohort (Anhui cohort; $\mathrm{n}=185$ ).

Results: Model A produced 7 predictors of unfavorable outcomes, while models B and C yielded 2 and 1 predictors, respectively. The areas under the curve (AUC) increased from 0.743 [model A; $95 \%$ confidence interval (CI): 0.680-0.782] to 0.889 (model A + B + C; 95\% CI: 0.851-0.916). The final prediction model included age, systolic blood pressure (SBP), body mass index (BMI), the Glasgow Coma Scale (GCS), the estimated glomerular filtration rate (eGFR), the early resumption of antithrombotic therapy, hematoma thickness and the presence of abdominal obesity, frailty and previous bleeding. Internal and external validation of the selected final model revealed adequate $\mathrm{C}$-statistics and calibration slope values (internal validation: 0.81 and 0.78 ; external validation: 0.80 and 0.76 , respectively).

Conclusions: This model provided a risk stratification tool to predict unfavorable outcomes in patients with recent AMI with antithrombotic-related CSDH. Because the study was based on ten readily practical and available variables, it may be widely applicable to guide management and complement clinical assessment. 
Keywords: Acute myocardial infarction (AMI); chronic subdural hematoma (CSDH); antithrombotic treatment; outcome prediction; risk stratification

Submitted Sep 02, 2020. Accepted for publication Nov 08, 2020.

doi: $10.21037 / \mathrm{cdt}-20-763$

View this article at: http://dx.doi.org/10.21037/cdt-20-763

\section{Introduction}

Chronic subdural hematoma (CSDH) is an increasingly common clinical condition in the aging population that is often due to head trauma (1). With the high prevalence of acute myocardial infarction (AMI), the use of antithrombotic (antiplatelet or anticoagulant) agents has increased over the last decade (2). Interestingly, CSDH in patients taking antithrombotic drugs appears to be an epidemic with increased healthcare costs (3). Approximately $40 \%$ of patients who experience CSDH receive antithrombotic agents at presentation (4). Emerging evidence has shown an association between the widely used antithrombotic drugs and a high risk of CSDH $(3,5)$. In such cases, close multidisciplinary teamwork is frequently confronted with the dilemma of comanaging CSDH and the ongoing requirement for antithrombotic agents. However, the clinical application of antithrombotic treatment in recent AMI patients with CSDH has been sparsely described, and a consensus identification method is not well clarified.

Although the patient prognosis of $\mathrm{CSDH}$ is highly variable, many factors have been proven to help risk stratification, including age, history of falls, female sex, minor head injury, epilepsy, hemodialysis, chronic alcohol use and low intracranial pressure states (6-8). Unfortunately, evidence linking these potential prognostic indexes to the occurrence of $\mathrm{CSDH}$ is limited to a few studies conducted among hospitalized patients with a history of previous trauma. Additionally, the predictive value of these indexes may differ from the possible strategy of antithrombotic treatment $(3,5)$. Patients with CSDH have a higher risk of recurrent thrombotic events (9). Importantly, the probability of adverse outcomes, such as cognitive decline, delirium, and speech impairment, is enormously elevated because of the occurrence of CSDH rebleeding $(1,3)$. For these CSDH patients on antithrombotic drugs, the lack of both finer selection criteria (optimal timing of recommencing antithrombotic drugs) other than the evaluated hematoma recurrence risk and thromboembolic events still affects the current treatment guidelines. These uncertainties make the best tailored strategy for antithrombotic agents in recent AMI patients with CSDH difficult. Accurate identification of the prognostic risk factors and outcome prediction would assist clinicians in counseling patients, tailoring therapy and stratifying patients for clinical trials. Several complex prognosis models are derived from different indexes (10-12), but the results are controversial and unsatisfactory.

Therefore, we explored and identified the prognostic indexes, and developed a clinically actionable model to predict subsequent outcomes in patients with recent AMI with antithrombotic-related CSDH. We present the following article in accordance with the TRIPOD reporting checklist (available at http://dx.doi.org/10.21037/cdt-20-763).

\section{Methods}

\section{Study population}

This retrospective study involved 553 patients with recent AMI with antithrombotic-related CSDH between April 2010 and August 2019 at three Chinese hospitals. Among these patients, 368 from the First Affiliated Hospital of Chengdu Medical College and the General Hospital of Western Theater Command were assigned to the training cohort (Chengdu training cohort; $\mathrm{n}=368$ ), and 185 from the Second Hospital of Anhui Medical University were separately used as an independent external validation cohort (Anhui external validation cohort; $\mathrm{n}=185$ ). According to the rule of overfitting of the regression model (13), we needed 50-100 antithrombotic-related CSDH episodes to assess 10 potential prognostic indexes. Assuming that the prevalence of recurrence $\mathrm{CSDH}$ was approximately $11.5-27 \%$ among patients with CSDH $(14,15)$, a sufficient sample size was at least 270 . To ensure an adequate number of events, we identified data from 368 individuals to develop the prognosis model. The inclusion criteria were as follows: age $\geq 18$ years, receiving standardized antithrombotic agents for AMI (16), and successfully undergoing standardized treatment of CSDH (e.g., evacuation of the hematoma) (14). The exclusion criteria are shown in Figure 1. All the patients 


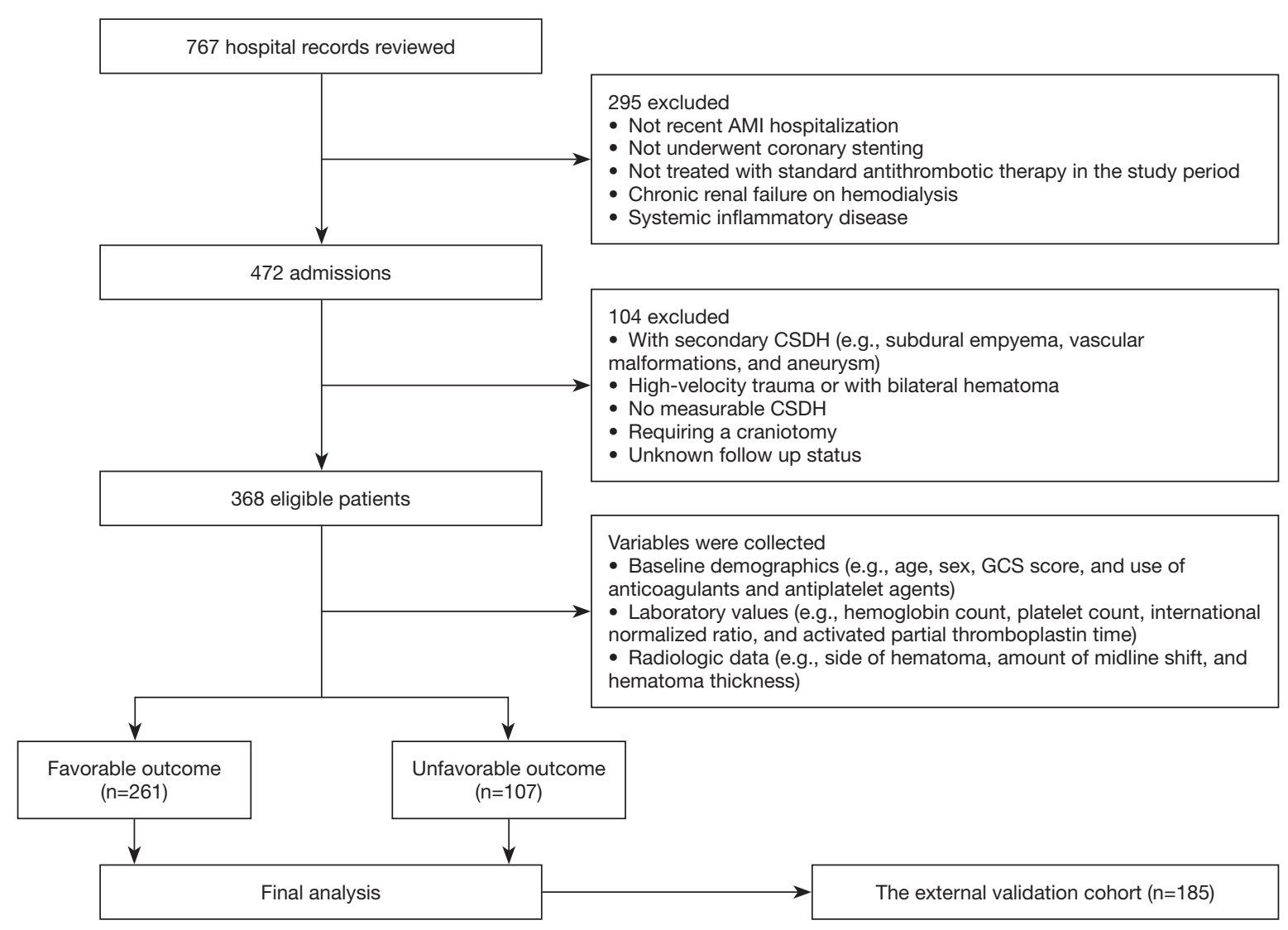

Figure 1 Study flow diagram. AMI, acute myocardial infarction; CSDH, chronic subdural hematoma; GCS, Glasgow Coma Scale.

provided informed consent. This study was approved by the research ethics board of the First Affiliated Hospital of Chengdu Medical College (No. CYYFYEC2010002), and was conducted in accordance with the Declaration of Helsinki (as revised in 2013).

\section{Variables}

Detailed clinical variables, such as the baseline demographics, laboratory values, and radiologic data were collected. Comorbidities were based on the conditions related to recent AMI with antithrombotic-related CSDH. The primary admission diagnoses of recent AMI with CSDH were defined by the International Classification of Diseases (ICD)-10 coding. Antithrombotic-related CSDH was defined as the first hospitalization that adopted longterm antithrombotic treatment and required hospitalization, regardless of any minor trauma (3). Abdominal obesity was defined as at least 1 of the following: waist circumference $\geq 102 \mathrm{~cm}$ or waist-to-hip (WHR) $\geq 0.90$ for men and waist circumference $\geq 88 \mathrm{~cm}$ or WHR $\geq 0.85$ for women (17). The Clinical Frailty Scale (CFS) was used to assess the patients' frailty based on their status before admission, and frailty was defined by a CFS score of 5 to 9 (18). We evaluated the timing of the resumption of antithrombotic therapy, including early resumption $(\leq 30$ days $)$ and late resumption (>30 days). Based on our collaborative multidisciplinary practice on the resumption of antithrombotics (Figure 2), postoperative patients were further classified as follows: (I) within the first 3 days, (II) between 4 and 14 days, (III) between 15 and 30 days, and (IV) more than 30 days.

\section{Outcome assessment}

All the subjects had at least 6 months of scheduled followup, which was generally determined by standardized telephone interviews, medical records or clinical visits. The primary outcome was a composite endpoint of either major adverse cardiovascular events (MACE), recurrence, or functional outcome such as the modified 

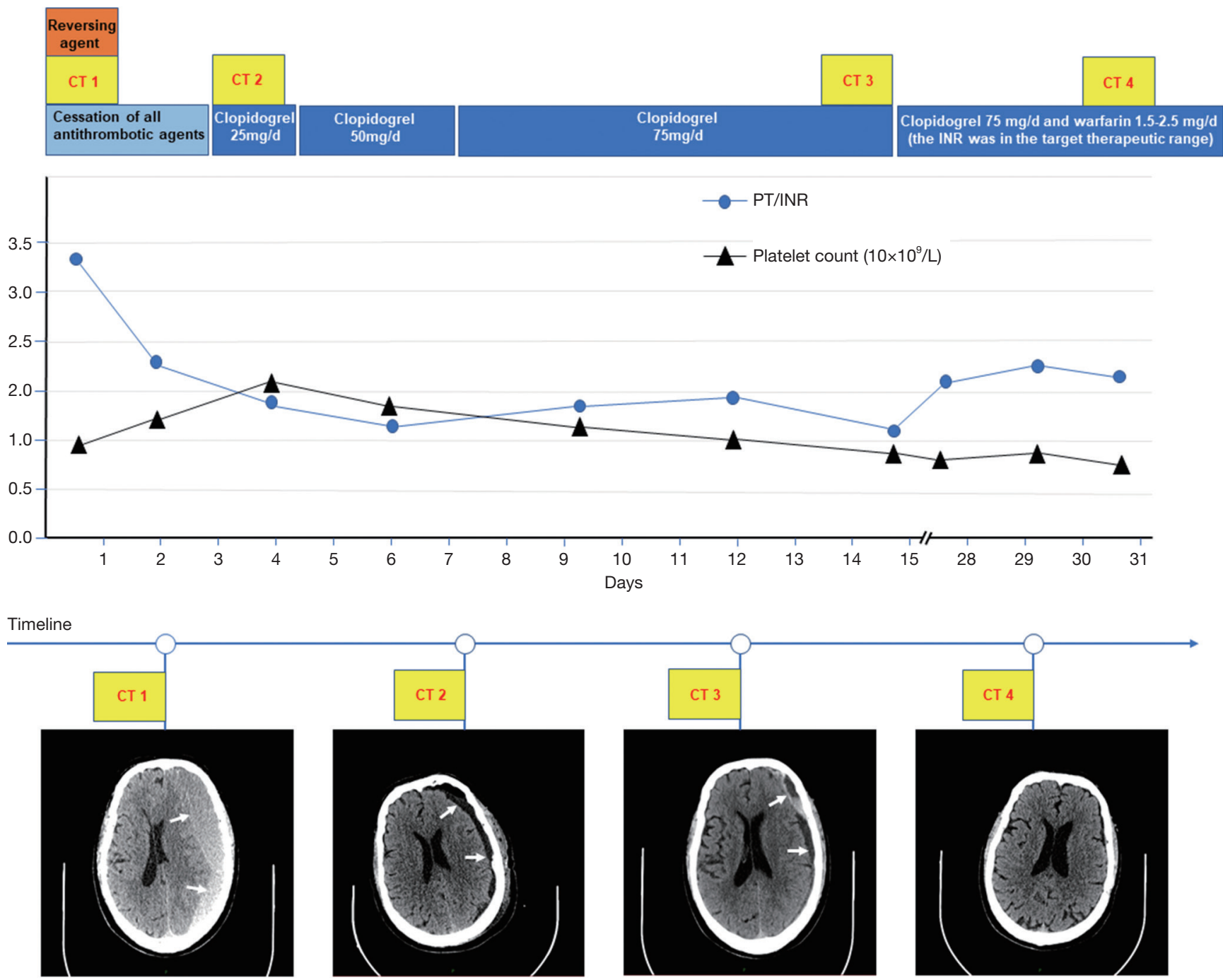

Figure 2 Examples of the patient's clinical course. Computed tomography (CT) sequence of the brain of a 76-year-old man with minor head injury 2 months after percutaneous coronary intervention. CT 1: chronic subdural hematoma (CSDH) (arrows) in the left frontotemporal occipital area 3 weeks after minor head injury. CT 2: subfrontal effusion and a gas shadow in the left frontal area, and no enlargement and resorption of the lesion (arrows) on day 3 after minimally invasive drainage for CSDH. CT 3: resorption of part of the lesion (arrows) on day 7 after minimally invasive drainage for CSDH. CT 4: follow-up head CT scanning 1 month later revealed the disappearance of the hematoma. Reversal of the antithrombotic treatment effect: platelet transfusion, fresh frozen plasma, clotting factors, or vitamin $\mathrm{K}$.

Rankin scale (mRS). MACEs were defined as a composite of cardiovascular mortality, MI, or target lesion revascularization (19). Recurrence was defined as any samesided recurrence of $\mathrm{CSDH}$ requiring reoperation within 6 months (20). The favorable outcome score was 0 to 1 , and the unfavorable outcome score was 2 to 6 , based on a dichotomized mRS score to the date of death or the last follow-up (12). Patients were divided into 2 groups (favorable and unfavorable outcomes) based on composite endpoints, and patients with unfavorable outcomes were defined if they had experienced at least 1 of the following: MACE, recurrence, or a mRS score of 2 to 6 .

\section{Model development and validation}

To develop a robust and valid prognosis prediction model, three approaches were performed: a demographic variable model (model A), a clinical marker model (model B) and 
a decision-driven model (model C). Model A was built on demographics and medical comorbidities, model B was built on laboratory results and radiologic data, and Model C was built on collaborative multidisciplinary practice on the resumption of antithrombotic therapy. A nomogram represented the final model. To evaluate both the discrimination and calibration of the nomogram in the validation cohort, a nomogram score for each individual was calculated.

\section{Statistical analysis}

Categorical variables were expressed as numbers and percentages using chi-square tests, and continuous variables were expressed as the mean \pm SD using Student's $t$-test. Univariate analyses were first used to determine possible predictors. Multiple logistic analysis with forward and stepwise selection procedures was then performed using significant factors from univariate analysis as inputs. The adjusted odds ratios (ORs) with $95 \%$ confidence intervals (CIs) were expressed as forest plots. To describe the discriminative performance, we used the receiver operating characteristic (ROC) curve with the corresponding $95 \%$ $\mathrm{CI}$ and computed areas under the curve (AUC). The Hosmer-Lemeshow test was used to assess the goodnessof-fit of the calibration of the models. To correct the overestimation, internal validation was performed using a bootstrap procedure with 1,000 bootstrap resamples. All statistical analyses were performed using SAS (version 9.4) and $\mathrm{R}$ (version 3.2.4). A $\mathrm{P}$ value $<0.05$ denoted statistical significance.

\section{Results}

\section{Patient characteristics}

The patient characteristics in the training cohort are provided in Table 1. Of the 368 included patients, 107 (29.1\%) patients had unfavorable outcomes. Compared with patients with a favorable outcome, those with an unfavorable outcome were significantly associated with the following parameters: older age, lower values of the median baseline Glasgow Coma Scale (GCS) and estimated glomerular filtration rate (eGFR), higher levels of systolic blood pressure (SBP) and hematoma thickness and the prevalence of abdominal obesity, as well as the presence of frailty and previous bleeding. In the favorable outcome group, most had adopted the early resumption of antithrombotics 4 to
14 days postoperatively, whereas none adopted the early resumption of antithrombotics 0 to 3 days postoperatively. Antithrombotic treatments of post-cessation in the training cohort are presented in Table 2.

\section{Predictors of outcomes}

Significant possible predictors (Table 1) determined by univariate analyses were further assessed by multiple logistic regression analysis (Figure 3). Multivariate analysis showed that age, SBP, body mass index (BMI), the GCS score, eGFR, the early resumption of antithrombotic therapy, hematoma thickness and the presence of abdominal obesity, frailty and previous bleeding were associated with higher odds of unfavorable outcomes.

\section{Prediction models}

The demographic variables model (model A) indicated that 7 candidate variables including age, SBP, BMI, the GCS score and the presence of abdominal obesity, frailty and previous bleeding were associated with unfavorable outcomes. The clinical marker model (model B) produced 2 variables, eGFR and hematoma thickness. The decision-driven model (model C) described the relationship between one variable based on early resumption and the risk of unfavorable outcomes. The discriminant abilities of model $\mathrm{A}$, model $\mathrm{A}+\mathrm{B}$, and model $\mathrm{A}$ $+\mathrm{B}+\mathrm{C}$ were analyzed using ROC curves, which indicated that the increase in predictors was associated with enhancing the discriminant ability of the prediction model (Figure 4). Specifically, the AUC increased from 0.743 (model A; $95 \%$ CI: $0.680-0.782$ ) to 0.889 (model A + B + C; $95 \%$ CI: $0.851-$ 0.916). As a further approximation, model $\mathrm{A}+\mathrm{B}+\mathrm{C}$ was the best prediction model using the final fitted multivariate model with 10 variables and is represented as a nomogram in Figure 5. The nomogram was well calibrated, as revealed by the calibration curves (Figure $6 A$ ), indicating that the final model had good performance and high applicability. With this final model, the C-statistic was 0.82 (95\% CI: 0.77 to 0.86 ), and the calibration slope was 0.92 (95\% CI: 0.35 to 1.42).

The associations between the final model with 10 variables and the risk of unfavorable outcome in the external validation cohort are shown in Table 3. Internal validation via a 1,000 -sample bootstrap of the final model revealed adequate $\mathrm{C}$-statistics and calibration slope values (C-statistics: $0.81,95 \%$ CI: 0.75 to 0.85 ; calibration slope: 
Table 1 Baseline characteristics of patients in the training cohort

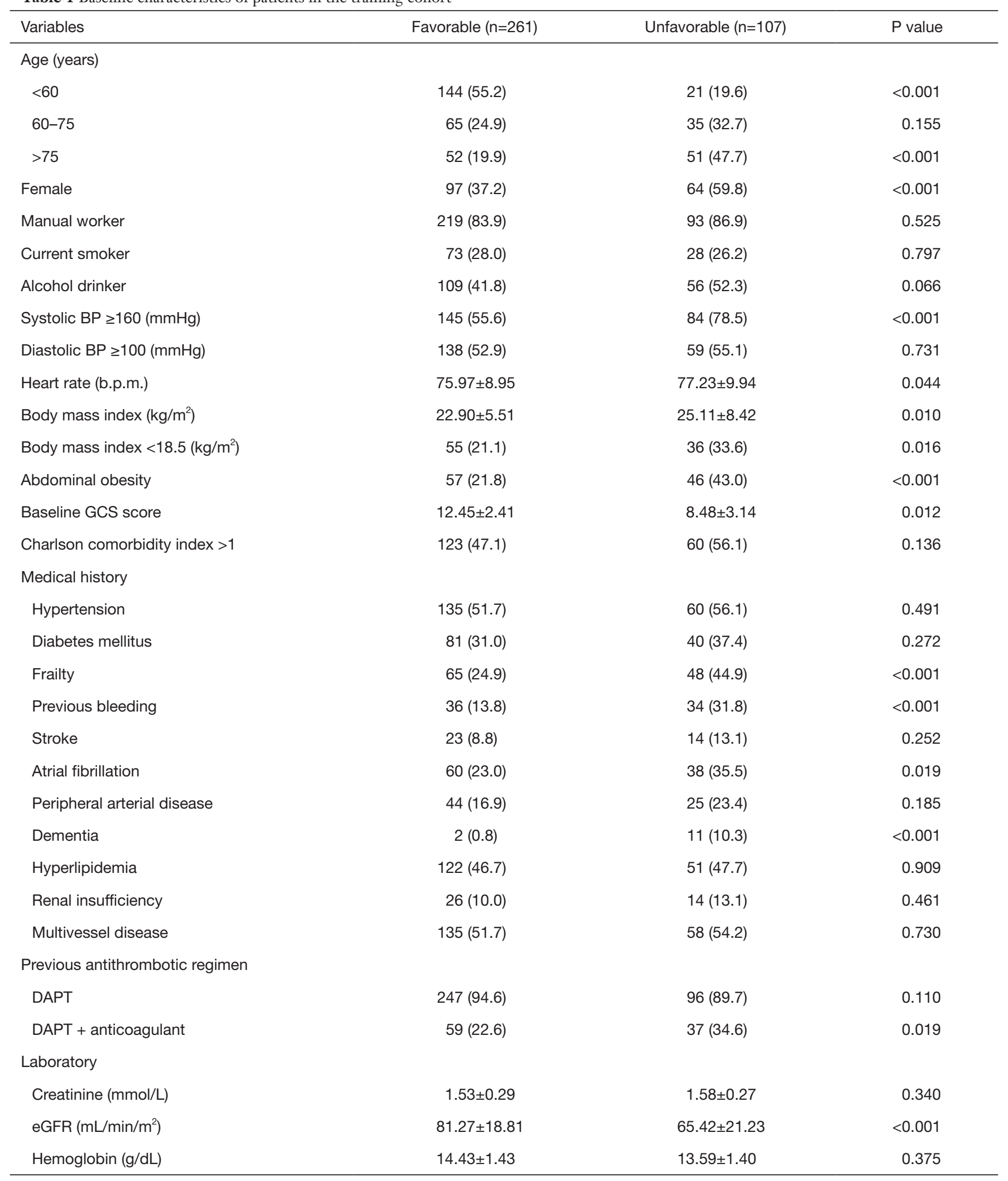

Table 1 (continued) 
Table 1 (continued)

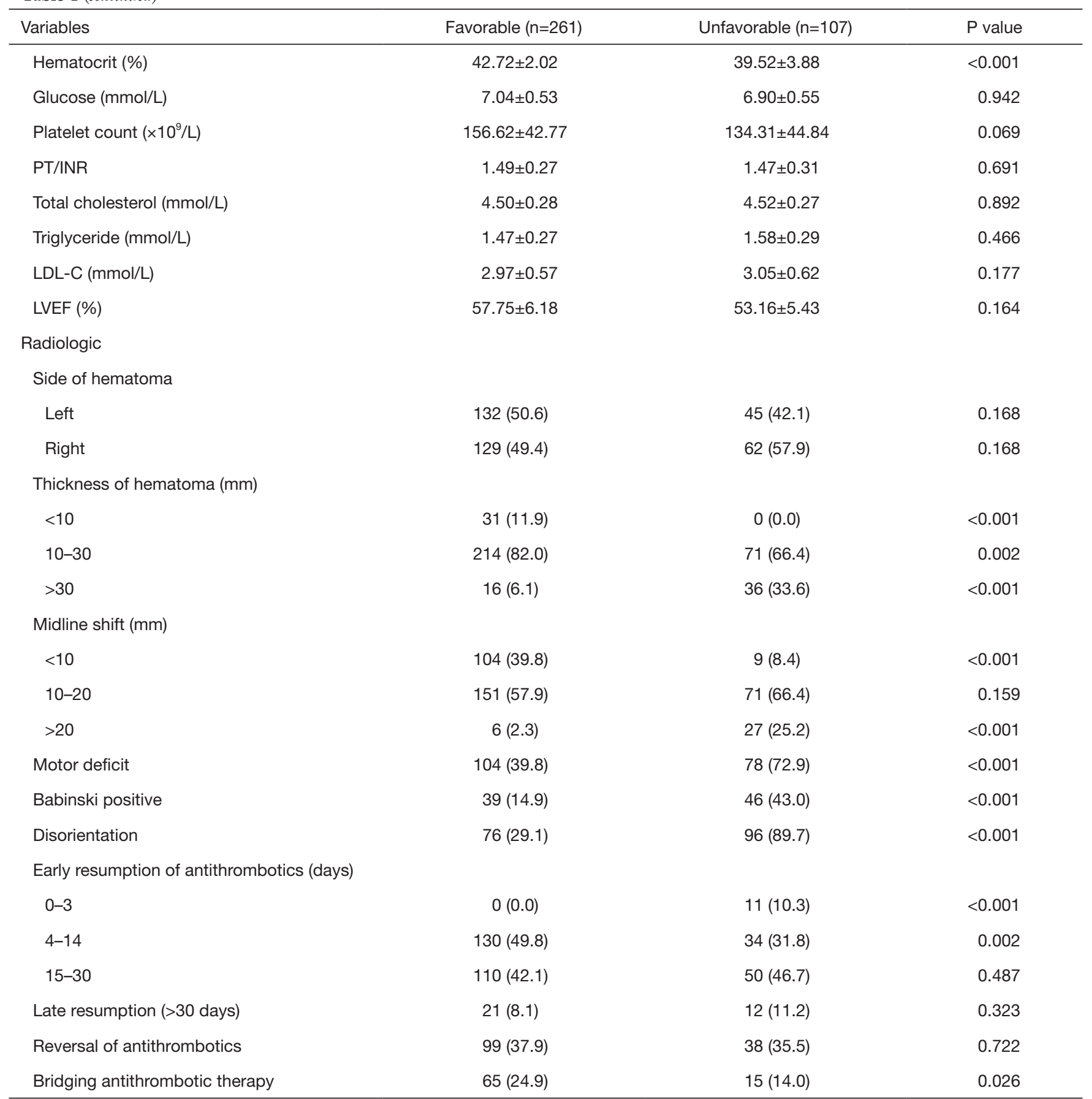

Values are expressed as the mean \pm SD or $n(\%)$. A P value $<0.05$ was considered statistically significant. BP, blood pressure; GCS, Glasgow Coma Scale; DAPT, dual antiplatelet therapy; eGFR, estimated glomerular filtration rate; PT/INR, prothrombin time/international normalized ratio; LDL-C, low-density lipoprotein cholesterol; LVEF, left ventricular ejection fraction.

0.78, 95\% CI: 0.53 to 1.09 ) (Figure $6 B$ ). Additionally, the C-statistic of the selected final model was 0.80 (95\% CI: 0.73 to 0.89 ) (Figure $6 C$ ) in the external validation cohort, with an optimism-corrected calibration slope of 0.76 (95\% CI: 0.50 to 1.02$)$, indicating a good level of discriminative ability. 
Table 2 Antithrombotic treatments post-cessation in the training cohort

\begin{tabular}{|c|c|c|c|c|}
\hline $\begin{array}{l}\text { Antithrombotic } \\
\text { regimen }\end{array}$ & $\begin{array}{l}\text { First antithrombotic } \\
\text { regimen within } 3 \text { days } \\
\text { post-cessation }{ }^{\mathrm{a}}(\mathrm{n}=368)\end{array}$ & $\begin{array}{l}\text { Antithrombotic regimen } \\
\text { days 4-14 post-cessation } \\
\qquad(n=357)\end{array}$ & $\begin{array}{l}\text { Antithrombotic regimen } \\
\text { days } 15-30 \text { post- } \\
\text { cessation }(n=193)\end{array}$ & $\begin{array}{l}\text { Antithrombotic regimen } \\
\text { more than } 30 \text { days post- } \\
\text { cessation }(n=33)\end{array}$ \\
\hline Aspirin & $0(0.0)$ & $0(0.0)$ & $4(2.0)$ & $0(0.0)$ \\
\hline Clopidogrel & $11(3.0)$ & $113(31.6)$ & $60(31.1)$ & $0(0.0)$ \\
\hline DAPT & $0(0.0)$ & $40(11.2)$ & $51(26.4)$ & $24(72.7)$ \\
\hline Aspirin + VKA & $0(0.0)$ & $0(0.0)$ & $6(3.1)$ & $0(0.0)$ \\
\hline Clopidogrel + VKA & $0(0.0)$ & $11(3.1)$ & $33(17.1)$ & 7 (21.2) \\
\hline DAPT+VKA & $0(0.0)$ & $0(0.0)$ & $3(1.6)$ & $2(6.1)$ \\
\hline
\end{tabular}

Values are expressed as $\mathrm{n}(\%) .{ }^{\text {a }}$, time from the cessation of antithrombotic treatments to prescription claim [days, mean (SD)]. VKAs, vitamin K antagonists; DAPT, dual antiplatelet therapy (aspirin + clopidogrel). Aspirin: $22.2 \pm 6.8$ days (median: 20 days). Clopidogrel: $17.5 \pm 14.3$ days (median: 13 days). VKAs: $25.7 \pm 7.8$ days (median: 22 days). Aspirin + clopidogrel (DAPT): $19.8 \pm 15.4$ days (median: 17 days). Aspirin + VKA: $24.1 \pm 6.8$ days (median: 23 days). Clopidogrel + VKA: $19.2 \pm 15.7$ days (median: 20 days). Triple therapy: $25.1 \pm 7.3$ days (median: 23 days).

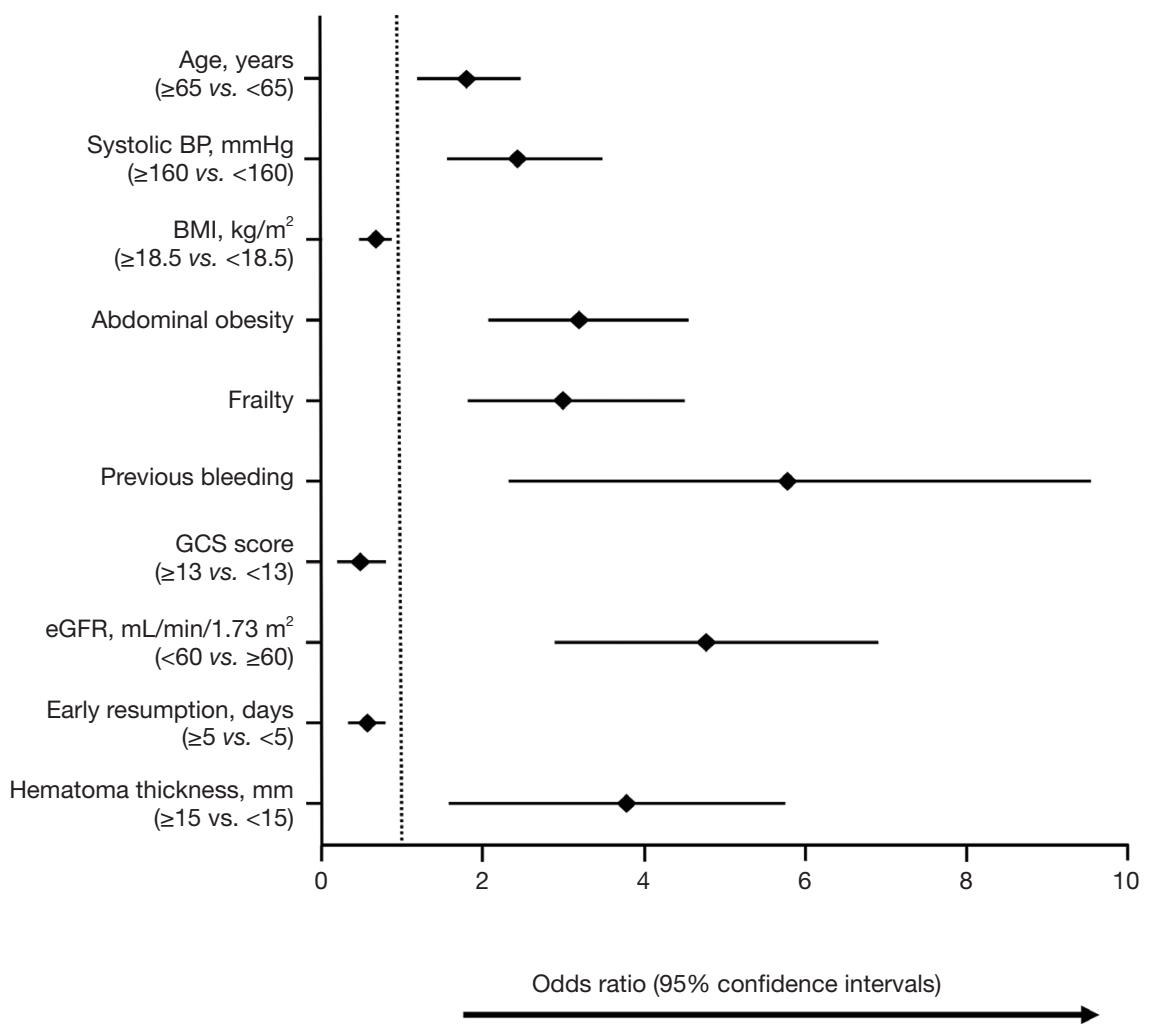

Unfavorable outcome

Figure 3 Forest plot depicting associations between age, systolic BP, BMI, GCS score, eGFR, early resumption, hematoma thickness and the presence of abdominal obesity, frailty and previous bleeding and the risk of unfavorable outcomes after antithrombotic-related CSDH in multivariable analysis. BP, blood pressure; BMI, body mass index; GCS, Glasgow Coma Scale; eGFR, estimated glomerular filtration rate; CSDH, chronic subdural hematoma. 


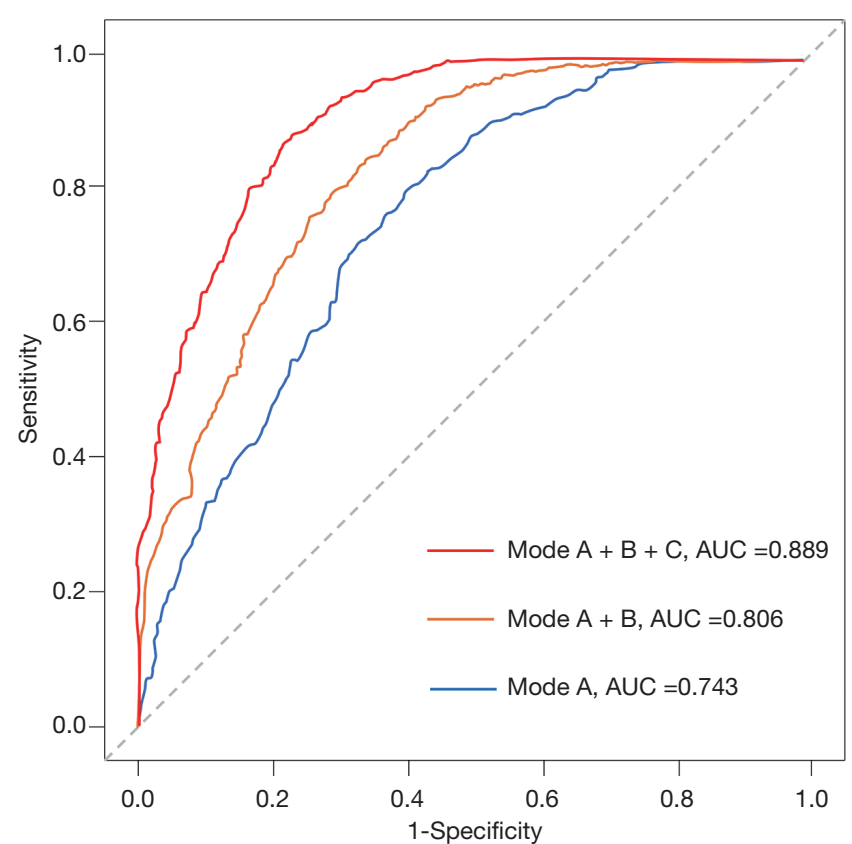

Figure 4 Receiver operating characteristic curves of each combinatorial prognosis model to predict the 6-month outcome. AUC, areas under the curve.

\section{Discussion}

This retrospective study demonstrates for the first time a deeper analysis of a scoring prognosis system combined with various risk factors to facilitate and improve outcome prediction among patients with recent AMI with antithrombotic-related CSDH. The main findings are as follows: (I) age, SBP, BMI, the GCS score, eGFR, early resumption, hematoma thickness and the presence of abdominal obesity, frailty and previous bleeding were independent predictors for unfavorable outcomes in patients with recent AMI with antithrombotic-related CSDH; (II) a scoring system for prognosticating patient outcomes after antithrombotic-related CSDH showed excellent discrimination and robust prediction; and (III) the scoring system was well calibrated in an external observational cohort of patients with recent AMI with antithromboticrelated $\mathrm{CSDH}$.

Few estimates of logical scoring systems exist to identify better guiding practice in patients with recent AMI with antithrombotic-related CSDH. Our results were confirmed and extended previous studies. First, we found that 10 variables from the final model (model $\mathrm{A}+\mathrm{B}+\mathrm{C}$ ) were associated with the risk of unfavorable outcomes among patients with recent AMI with antithrombotic-related
$\mathrm{CSDH}$. Some of these variables were consistent with previous multivariate analysis, such as age, female sex, low BMI, the GCS score, hematoma thickness and the presence of previous bleeding (10-12,21). The major mechanisms linking the six above mentioned variables with CSDH are relatively well elaborated in existing knowledge (10-12, 14,15,21). Similarly, Kwon et al. (11) devised a scoring system regarding the prognosis of patients with $\mathrm{CSDH}$ that can assist in the necessity of surgery; however, the authors did not focus on the population with recent AMI receiving antithrombotic agents.

Second, the fear of falls and subsequent CSDH are common reasons to avoid initiating antithrombotic therapy or discontinuing therapy in patients with recent AMI $(3,5)$. The main principles of antithrombotic strategy in this complex setting need to prevent stent thrombosis while avoiding increased bleeding risk by the safely tailored use of antiplatelet therapy (22). Upon a bleeding episode, potential strategies include downgrading from triple to dual therapy, stopping DAPT or all antithrombotic agents transitorily, shortening DAPT duration, and reversal until bleeding has stopped unless the thrombotic risk is prohibitive, or even resumption with a single antiplatelet drug (preferably with clopidogrel) when bleeding has stopped $(19,23,24)$, findings that were consistent with our model $\mathrm{C}$ analysis based on collaborative multidisciplinary practice. Life-threatening bleeding is the only absolute indication to stop and reverse antithrombotic therapy $(19,23)$. The best known WOEST trial showed that double therapy (clopidogrel plus vitamin $\mathrm{K}$ antagonist) was safer regarding hemorrhage than triple therapy, and simultaneous registries have demonstrated similar findings (25). Given the different ethnicities of Asian patients, 3 main bleeding risk score models exist: the CRUSADE, ACTION, and ACUITY-HORIZONS models (26-28). The CRUSADE and ACTION models hold similar baseline factors, including the heart rate, SBP, heart failure, diabetes, and vascular disease, whereas the ACUITY-HORIZONS model did not have these factors and instead used the variable treatment with the antithrombotic regimen (26-28). However, our final model did not include these variables, mainly due to the population confined to the clinical settings in antithrombotic-related CSDH. For the resumption of antiplatelet therapy, evidence appears to reveal no difference between early and late resumption $(3,5,29)$. Our study suggested that the resumption of a single antithrombotic more than 3 days postoperatively may be safe.

Third, BMI significantly influences the association 


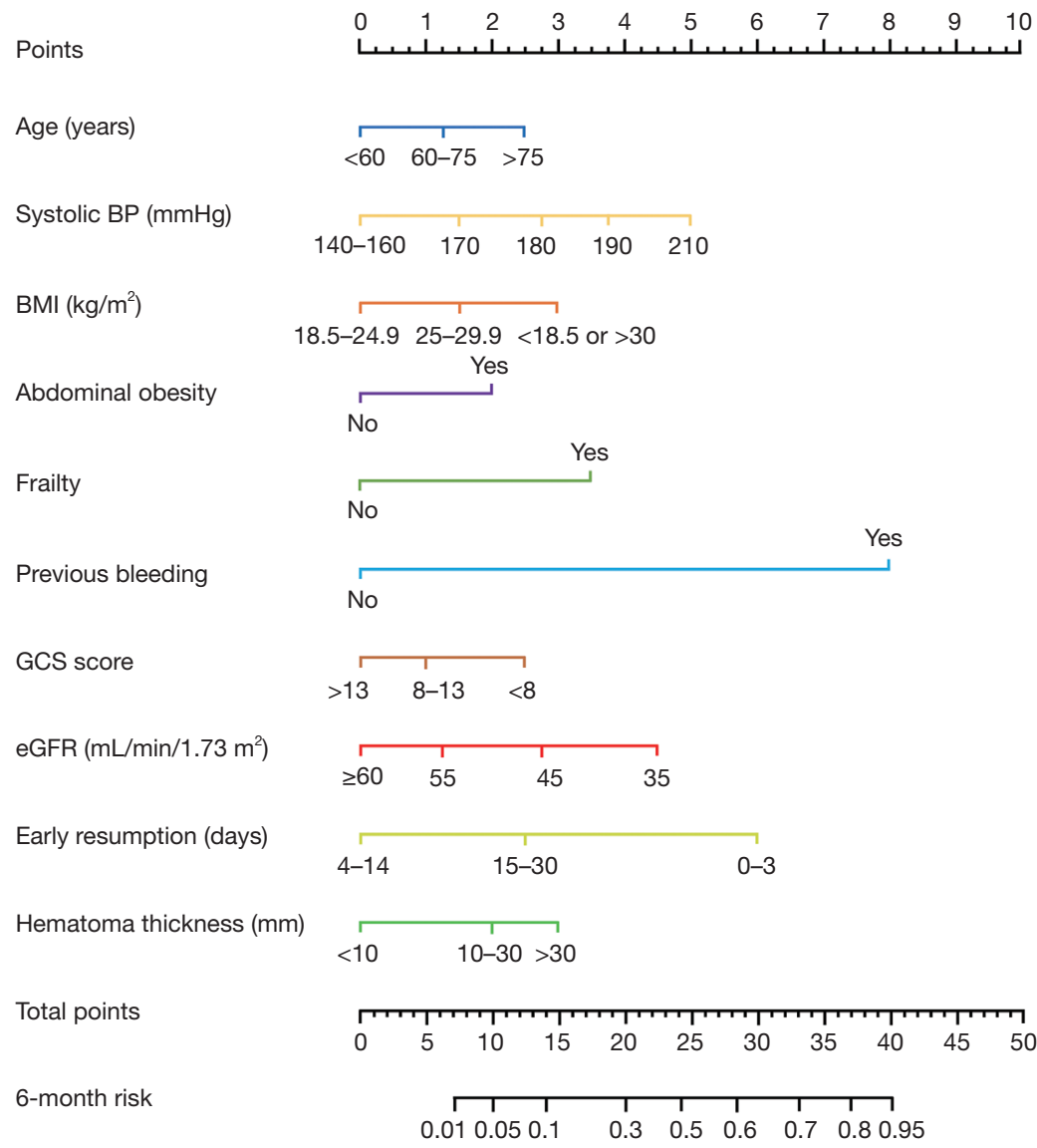

Figure 5 Nomogram for the final model developed in the training cohort. Each variable corresponds to a point (top). These points are then summed to translate into a 6-month risk of an unfavorable outcome (bottom). BP, blood pressure; BMI, body mass index; GCS, Glasgow Coma Scale; eGFR, estimated glomerular filtration rate.

between clopidogrel therapy and the CYP2C19 genotype, and extreme BMI (low and high) predicts the escalation of the bleeding risk (30). However, BMI does not accurately describe body fat accumulation, and surrogate markers of abdominal obesity, such as WHR or waistline circumference, might be more important predictors for unfavorable outcomes in patients with recent AMI with antithrombotic-related CSDH. In our study, the association between abdominal obesity and unfavorable outcomes may be explained by these mechanisms, such as impaired suppression of adipocyte lipolysis, elevated C-reactive protein levels, elevated nonesterified fatty acid levels, increased macrophage-related atherogenic cytokines, and low-grade systemic inflammation, potentially leading to low-grade systemic inflammation and vascular endothelial dysfunction, which contribute to increased cardiovascular risk $(17,31,32)$.
Fourth, our findings support previous observations showing the association of decreased renal function with a higher bleeding risk (33). The CHADS2 score also recommends that decreased renal function is associated with the risk of stroke, possibly because all the direct oral anticoagulants are eliminated via the kidney (34). Despite crucial prior steps in the antithrombotic therapy of AMI patients with decreased renal function, including the DAPT, PARIS, and PRECISE-DAPT scores, many uncertainties persist (33). Further complications arise when patients with decreased renal function have a very high risk of impaired antiplatelet effects by clopidogrel and aspirin $(34,35)$.

Fifth, one notable finding in the multivariate analysis was that frailty was independently associated with the primary composite outcome. Frailty is an emerging predictor in cardiovascular disease revealing increased disease vulnerability and decreased physiological reserves, which 

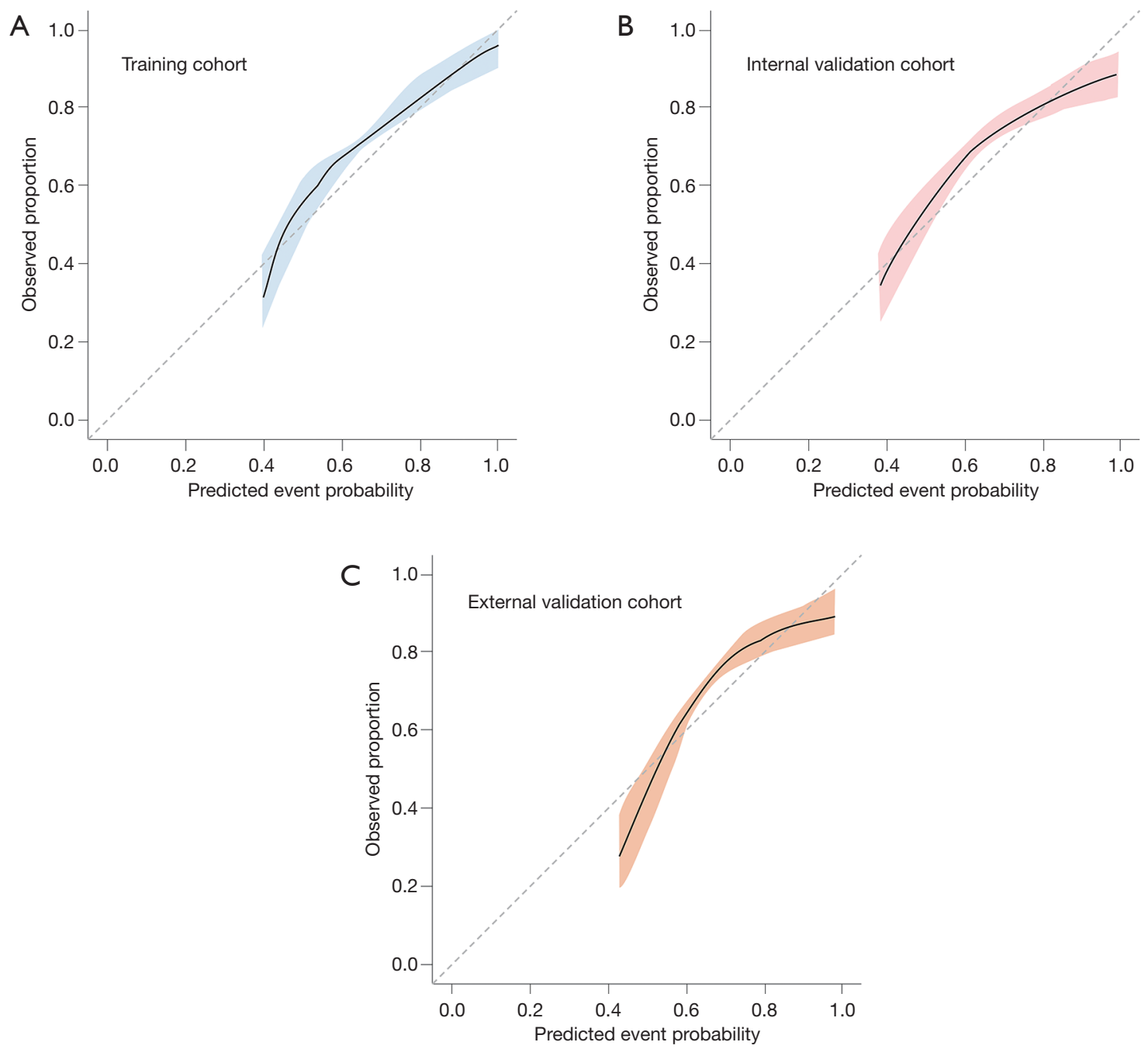

Figure 6 Calibration curves for the nomogram in the training (A), internal validation (B) and external validation (C) cohorts. Calibration curves indicate the goodness-of-fit of the nomogram. The $45^{\circ}$ gray line represents the ideal prediction.

transform the drug kinetics and toxicity of standard doses of antithrombotics $(18,36)$. Numerous previous studies have described that frailty is strongly associated with mortality, and approximately $20-40 \%$ of patients with cardiac surgery were either fragile or at risk for frailty $(18,36)$. Consistently, in CSDH patients, Shimizu et al. (37) observed a poorer prognosis in patients with frailty than those in without frailty. Thus, few recent trials on antithrombotic treatment have evaluated frail patients to help guide decision-making.

To the best of our knowledge, this study is the first to devise an objective, simple, numeric, and visual version of a prognostic model with respect to patients with recent AMI with antithrombotic-related CSDH. Using a retrospective study to identify the possible role of numerous clinical variables and biomarkers in the clinical setting, a nomogram incorporating factors of the antithrombotic regimen was established and emerged as being more effective than a single clinical prognostic index. Importantly, the validation cohort at another hospital was exploited to validate the effectiveness of the current nomogram and achieve a high C-index. Another strength of our study approach is that it confirms that all the indexes are intuitive to clinicians, indicating they are feasible and common in routine hospital settings. This tool can be effective in risk assessment on an individual level to identify who may merit intensive screening and/or targeted prevention strategies (e.g., randomized primary prevention trials) to reduce the burden of CSDH patients with recent AMI. This risk 
Table 3 Baseline characteristics of patients in the external validation cohort

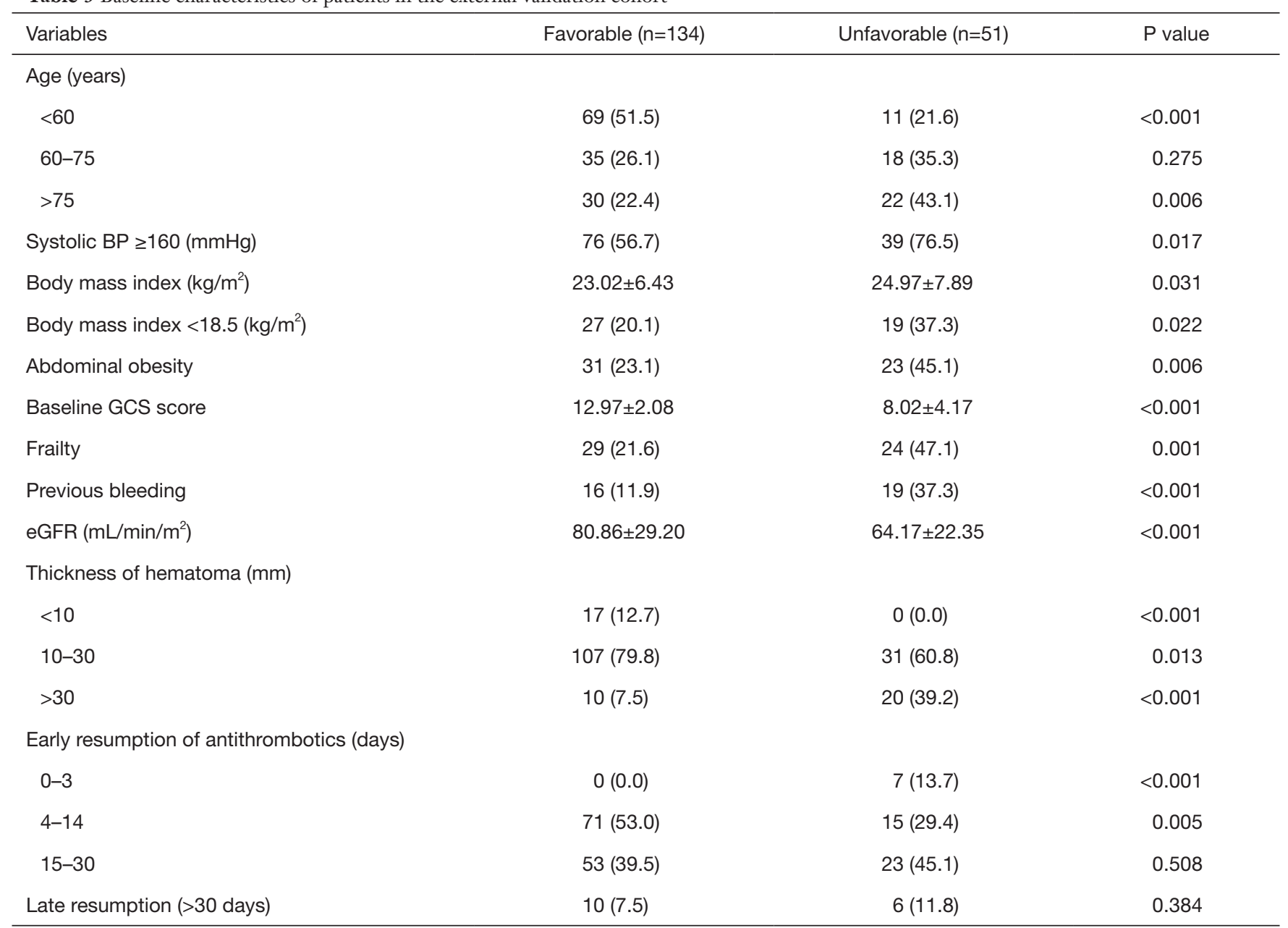

Values are expressed as the mean \pm SD or $n(\%)$. A P value $<0.05$ was considered statistically significant. BP, blood pressure; GCS, Glasgow Coma Scale; eGFR, estimated glomerular filtration rate.

score is a reliable and appealing screening tool to identify populations in the primary care setting who are at higher risk to develop of CSDH. Sequential screening for CSDH patients with recent AMI could integrate traditional and novel biomarkers (e.g., BMI, eGFR, and SBP) and collaborative multidisciplinary practice (e.g., resumption of antithrombotic therapy) in a stepwise, individualized, and cost-effective approach.

\section{Limitations}

Several limitations deserve mention. First, this was a retrospective study, and potential residual confounders might be present. There is scant evidence to guide the ideal timing to recommend antithrombotic agents, and information on the type and dose of oral antithrombotic agents is complex $(3,5,19)$. The sample size of patients using novel oral anticoagulants (NOACs) was relatively small; thus, these results may not be generalizable for patients on NOACs. Therefore, our observed findings might be conservative. Regardless, the model performed robustly as well as expected in an external validation cohort. Second, although the prognostic model can support clinicians in effectively and accurately predicting outcomes, it cannot completely replace the best tailored strategy made by clinicians. Third, it is emphasized that a scoring system might not capture all the existing predictors, because of the clinical data from three hospital databases. However, this consensus limitation is deemed minor, and all patients' data on vital status were complete. Fourth, the validation 
cohort reviewed only patients in China, limiting the generalizability to different populations and adherence. Finally, an unfavorable outcome was defined as a composite endpoint of MACE, recurrence, or a mRS score of 2 to 6 . The subgroup cohorts according to the above mentioned three different endpoints preclude multivariate analyses because of the small patient number in each subgroup. Accordingly, a large sample size and a prospective multicenter study design are needed in future studies.

\section{Conclusions}

Our study provides further evidence supporting the prognostic value of several practical variables for the outcomes of antithrombotic-related CSDH in patients with recent AMI. It may serve as a user-friendly tool tailored to the vital status of each individual, facilitating risk stratification, complementing clinical assessment, and tailoring treatment strategies during clinical decision making.

\section{Acknowledgments}

The authors thank all the colleagues who cooperated with them.

Funding: This research was supported by grants from the National Natural Science Foundation of China (81970262) (PW), Innovation Team Project Department of Education of Sichuan Province (18TD0030) (PW), Special Funding for the Central Government to Guide Local Science and Technology Development of Sichuan Province (2020ZYD036) (P.W.), and grants from the Scientific Research Fund of Chengdu Medical College (CYZYB20-07) (JW).

\section{Footnote}

Reporting Checklist: The authors have completed the TRIPOD reporting checklist. Available at http://dx.doi. org/10.21037/cdt-20-763

Data Sharing Statement: Available at http://dx.doi. org/10.21037/cdt-20-763

Conflicts of Interest: All authors have completed the ICMJE uniform disclosure form (available at http://dx.doi. org/10.21037/cdt-20-763). The authors have no conflicts of interest to declare.
Ethical Statement: The authors are accountable for all aspects of the work in ensuring that questions related to the accuracy or integrity of any part of the work are appropriately investigated and resolved. This study was approved by the research ethics board of the First Affiliated Hospital of Chengdu Medical College (No. CYYFYEC2010002), and was conducted in accordance with the Declaration of Helsinki (as revised in 2013). All patients provided informed consent by the local institutional review boards.

Open Access Statement: This is an Open Access article distributed in accordance with the Creative Commons Attribution-NonCommercial-NoDerivs 4.0 International License (CC BY-NC-ND 4.0), which permits the noncommercial replication and distribution of the article with the strict proviso that no changes or edits are made and the original work is properly cited (including links to both the formal publication through the relevant DOI and the license). See: https://creativecommons.org/licenses/by-nc-nd/4.0/.

\section{References}

1. Jiang R, Zhao S, Wang R, et al. Safety and efficacy of atorvastatin for chronic subdural hematoma in Chinese patients: A Randomized Clinical Trial. JAMA Neurol 2018;75:1338-46.

2. Hausenloy DJ, Kharbanda RK, Møller UK, et al. Effect of remote ischaemic conditioning on clinical outcomes in patients with acute myocardial infarction (CONDI-2/ ERIC-PPCI): a single-blind randomised controlled trial. Lancet 2019;394:1415-24.

3. Nathan S, Goodarzi Z, Jette N, et al. Anticoagulant and antiplatelet use in seniors with chronic subdural hematoma: systematic review. Neurology 2017;88:1889-93.

4. Poon MTC, Rea C, Kolias AG, et al. Influence of antiplatelet and anticoagulant drug use on outcomes after chronic subdural hematoma drainage. J Neurotrauma 2019. [Epub ahead of print].

5. Gaist D, García Rodríguez LA, Hellfritzsch M, et al. Association of antithrombotic drug use with subdural hematoma risk. JAMA 2017;317:836-46.

6. Han MH, Ryu JI, Kim CH, et al. Predictive factors for recurrence and clinical outcomes in patients with chronic subdural hematoma. J Neurosurg 2017;127:1117-25.

7. Motiei-Langroudi R, Stippler M, Shi S, et al. Factors predicting reoperation of chronic subdural hematoma following primary surgical evacuation. J Neurosurg 
2018;129:1143-50.

8. Zanaty M, Park BJ, Seaman SC, et al. Predicting chronic subdural hematoma recurrence and stroke outcomes while withholding antiplatelet and anticoagulant agents. Front Neurol 2020;10:1401-27.

9. Murthy SB, Wu X, Diaz I, et al. Non-traumatic subdural hemorrhage and risk of arterial ischemic events. Stroke 2020;51:1464-69.

10. Stanišic M, Pripp AH. A reliable grading system for prediction of chronic subdural hematoma recurrence requiring reoperation after initial burr-hole surgery. Neurosurgery 2017;81:752-60.

11. Kwon CS, Al-Awar O, Richards O, et al. Predicting prognosis of patients with chronic subdural hematoma: a new scoring system. World Neurosurg 2018;109:e707-14.

12. Yan C, Yang M, Huang Y. A reliable nomogram model to predict the recurrence of chronic subdural hematoma after burr hole surgery. World Neurosurg 2018;118:e356-66.

13. Vittinghoff E, McCulloch CE. Relaxing the rule of ten events per variable in logistic and Cox regression. Am J Epidemiol 2007;165:710-8.

14. Kolias AG, Chari A, Santarius T, et al. Chronic subdural haematoma: modern management and emerging therapies. Nat Rev Neurol 2014;10:570-8.

15. Schucht P, Fischer U, Fung C, et al. Follow-up computed tomography after evacuation of chronic subdural hematoma. N Engl J Med 2019;380:1186-7.

16. Neumann FJ, Sousa-Uva M, Ahlsson A, et al. 2018 ESC/ EACTS guidelines on myocardial revascularization. Eur Heart J 2019;40:87-165.

17. Wan J, Zhou P, Wang D, et al. Impact of normal weight central obesity on clinical outcomes in male patients with premature acute coronary syndrome. Angiology 2019;70:960-8.

18. Shimura T, Yamamoto M, Kano S, et al. Impact of the clinical frailty scale on outcomes after transcatheter aortic valve replacement. Circulation 2017;135:2013-24.

19. Nabi H, Rørth R, Tajchman DH, et al. Antithrombotic treatment and major adverse cardiac events after bleeding in patients with myocardial infarction: a retrospective analysis of nationwide registry data. Eur Heart J Cardiovasc Pharmacother 2020;6:14-21.

20. Licci M, Kamenova M, Guzman R, et al. Influence of postoperative thrombosis prophylaxis on the recurrence of chronic subdural hematoma after burr-hole drainage. Crit Care Med 2018;46:e26-32.

21. Wan J, Wang P, Zhou P, et al. Predictors and management of antiplatelet-related bleeding complications for acute coronary syndrome in Chinese elderly patients. Cell Physiol Biochem 2018;50:1164-77.

22. Li L, Geraghty OC, Mehta Z, et al. Age-specific risks, severity, time course, and outcome of bleeding on longterm antiplatelet treatment after vascular events: a population-based cohort study. Lancet 2017;390:490-9.

23. Halvorsen S, Storey RF, Rocca B, et al. Management of antithrombotic therapy after bleeding in patients with coronary artery disease and/or atrial fibrillation: expert consensus paper of the European Society of Cardiology Working Group on Thrombosis. Eur Heart J 2017;38:1455-62.

24. Hahn JY, Song YB, Oh JH, et al. Effect of P2Y12 inhibitor monotherapy vs dual antiplatelet therapy on cardiovascular events in patients undergoing percutaneous coronary intervention: the SMART-CHOICE randomized clinical trial. JAMA 2019;321:2428-37.

25. Dewilde WJ, Oirbans T, Verheugt FW, et al. Use of clopidogrel with or without aspirin in patients taking oral anticoagulant therapy and undergoing percutaneous coronary intervention: an open-label, randomized, controlled trial. Lancet 2013;381:1107-15.

26. Mathews R, Chen AY, Thomas L, et al. Differences in short-term versus long-term outcomes of older black versus white patients with myocardial infarction: findings from the Can Rapid Risk Stratification of Unstable Angina Patients Suppress Adverse Outcomes with Early Implementation of American College of Cardiology/ American Heart Association Guidelines (CRUSADE). Circulation 2014;130:659-67.

27. Desai NR, Kennedy KF, Cohen DJ, et al. Contemporary risk model for in-hospital major bleeding for patients with acute myocardial infarction: The Acute Coronary Treatment and Intervention Outcomes Network (ACTION) registry®-Get With The Guidelines (GWTG)®. Am Heart J 2017;194:16-24.

28. Mehran R, Pocock SJ, Nikolsky E, et al. A risk score to predict bleeding in patients with acute coronary syndromes. J Am Coll Cardiol 2010;55:2556-66.

29. Fornebo I, Sjåvik K, Alibeck M, et al. Role of antithrombotic therapy in the risk of hematoma recurrence and thromboembolism after chronic subdural hematoma evacuation: a population-based consecutive cohort study. Acta Neurochir (Wien) 2017;159:2045-52.

30. Mo J, Chen Z, Xu J, et al. Efficacy of clopidogrel-aspirin therapy for stroke does not exist in C YP2C19 loss-offunction allele noncarriers with overweight/obesity. Stroke 2020;51:224-31. 
31. Lee SR, Choi EK, Park CS, et al. Direct oral anticoagulants in patients with nonvalvular atrial fibrillation and low body weight. J Am Coll Cardiol 2019;73:919-31.

32. Choi D, Choi S, Son JS, et al. Impact of discrepancies in general and abdominal obesity on major adverse cardiac events. J Am Heart Assoc 2019;8:e013471.

33. Covic A, Genovesi S, Rossignol P, et al. Practical issues in clinical scenarios involving CKD patients requiring antithrombotic therapy in light of the 2017 ESC guideline recommendations. BMC Med 2018;16:158-98.

34. Burlacu A, Genovesi S, Goldsmith D, et al. Bleeding in advanced $\mathrm{CKD}$ patients on antithrombotic medication a

Cite this article as: Zhou P, Wan J, Ran F, Gao F, Yang D, Dai X, Sun Y, Wang P. Development and validation of a prognostic prediction model for antithrombotic-related chronic subdural hematoma in patients with recent acute myocardial infarction. Cardiovasc Diagn Ther 2020;10(6):1770-1784. doi: 10.21037/ cdt-20-763 critical appraisal. Pharmacol Res 2018;129:535-43.

35. Proietti R, Gonzini L, Pizzimenti G, et al. Glomerular filtration rate: A prognostic marker in atrial fibrillation-A subanalysis of the AntiThrombotic Agents Atrial Fibrillation. Clin Cardiol 2018;41:1570-7.

36. Ekerstad N, Swahn E, Janzon M, et al. Frailty is independently associated with short-term outcomes for elderly patients with non-ST-segment elevation myocardial infarction. Circulation 2011;124:2397-404.

37. Shimizu K, Sadatomo T, Hara T, et al. Importance of frailty evaluation in the prediction of the prognosis of patients with chronic subdural hematoma. Geriatr Gerontol Int 2018;18:1173-6. 